\title{
大气等离子体喷涂的单片层研究进展
}

\author{
李大川 ${ }^{1,2}$, 赵华玉 ${ }^{1}$, 钟兴华 ${ }^{1}$, 陶顺衍 ${ }^{1}$
}

(1. 中国科学院 上海硅酸盐研究所, 中国科学院特种无机涂层重点实验室, 上海 201899; 2. 中国科学院大学, 北 京 100049)

摘 要: 大气等离子体喷涂技术是一种常用的涂层制备工艺。作为所制备涂层的结构基元，单片层的形貌特征及堆 叠行为决定了涂层的微结构, 对涂层性能产生显著影响。本文较为系统地综述了与熔滴自身理化状态相关的主体因 素、与沉积涂层的祄底相关的客体因素以及环境因素等对单片层形成过程的影响, 着重分析了粉体尺寸、补底预热 过程等产生的多种不同影响及其之间的关联性, 并通过对所述文献涉及实验方法各自特点的比较, 提出未来的实 验和模拟研究或都将向着与真实生产条件更加接近的方向发展。

关 键 词: 大气等离子体喷涂; 热障涂层; 熔滴; 单片层; 综述

中图分类号: TQ174 文献标识码: A

\section{Research Progresses of Atmospheric Plasma Sprayed Splat}

\author{
LI Da-Chuan $^{1,2}$, ZHAO Hua-Yu ${ }^{1}$, ZHONG Xing-Hua ${ }^{1}$, TAO Shun-Yan ${ }^{1}$
}

(1. Key Laboratory of Inorganic Coating Materials CAS, Shanghai Institute of Ceramics, Chinese Academy of Sciences, Shanghai 201899, China; 2. University of Chinese Academy of Sciences, Beijing 100049, China)

\begin{abstract}
Atmospheric plasma spraying (APS) is a well-established method to fabricate coatings. Since the coatings are piled up with splat, the morphology of splats is of significant importance for the microstructure and performance of coatings deposited by APS. In the current review, the influences of parameters in spraying processes on formation of splats were divided into three categories, namely the droplet-related factors, substrate-related factors and environmental-related factors. The influence of above mentioned factors on morphology of splats were systematically introduced. Among them, effect of powder size and substrates preheating were emphatically discussed. Future research direction was also pointed out based on the reviewed literature in this paper.
\end{abstract}

Key words: atmospheric plasma spraying; thermal barrier coatings; droplet; splat; review

随着工业技术的不断发展，对零部件综合性能 的要求越来越高, 表面工程的重要性日益突出。在 零部件表面制备厚度数微米到数毫米的功能薄层, 可以改变零部件的表面性状, 大幅提升其性能, 进 而有效延长其使用寿命, 这符合绿色、循环经济和 可持续发展的要求 ${ }^{[1-2]}$ 。热喷涂(thermal spraying)是 表面工程的重要组成部分, 从1910年诞生之初的固
定式坩埚熔融喷射装置发展至今, 喷涂工艺不断演 化，对喷涂过程的在线监测和控制技术也迅速发展， 喷涂材料种类也不断扩展，形成了包括设备、原 料、工艺及应用的完整工业体系 ${ }^{[3-4]}$ 。据不完全统计, 2014 年我国热喷涂行业的总产值约为 120 亿元, 在航 空航天、高端装备制造、微电子、国防、汽车、轨 道交通、海洋装备等产业都已取得较好的应用 ${ }^{[5]}$ 。

收稿日期：2016-08-08; 收到修改稿日期：2016-09-26

基金项目：上海市经信委军民结合专项项目(JMJH2014053)

Special Project for Army-Civilian Combination of Shanghai Municipal Commission of Economy and Informatization (JMJH2014053)

作者简介: 李大川(1987-), 男，博士研究生. E-mail: dachuan_li@student.sic.ac.cn

通讯作者：陶顺衍，研究员. E-mail: sytao@mail.sic.ac.cn 
大气等离子体喷涂(atmospheric plasma spraying, APS) 是热喷涂技术的一种, 诞生于上世纪五十年代 中期 ${ }^{[6]}$, 其特征是以高温高速的等离子体射流为热 源, 高温区温度可超过 $8000 \mathrm{~K}$, 可以熔化所有固态 物质 ${ }^{[7]}$, 因而在制备陶瓷涂层方面具有独特的优 势。在大气等离子体喷涂工艺中, 粉末颗粒被载气 送入高温高速的等离子体焰流, 经加热和加速, 以 熔融或半熔融的状态高速撞击衬底, 迅速铺展并冷 却固化, 最终形成扁平的单片层(splat); 大量单片 层不断堆叠最终形成宏观尺度的涂层。

作为大气等离子体喷涂涂层的特征单元, 单片 层的形貌特征及单片层之间的堆叠行为决定了涂层 的微结构。以单片层为主要对象的应用基础研究的 意义可见一斑。公开发表的论文中专注于单片层形 成过程的实验和模拟研究数量有限, 但有关涂层的 微结构或性能的研究几乎都会以这些基础研究为依 据。单片层相关研究主要集中在法国、美国、德国 和日本等, 国内的西安交通大学和中国科学院上海 硅酸盐研究所等在这一领域也做出了一定贡献。

本文通过分析单片层研究中的部分成果, 将影 响单片层形成的因素分为三类, 着重介绍其中与粉 体/熔滴自身有关的主体因素以及与祄底有关的客 体因素, 并展望了未来研究工作的发展方向。

\section{1 喷涂的主体: 粉体}

\section{1 粉体化学组成}

大气等离子体喷涂技术由于具有焰流温度高、 喷涂环境中含氧等特点, 特别适于氧化物涂层的高 效制备, 其中较为常见的有氧化铝、氧化钛、氧化 铬、羟基磷灰石、氧化锆、氧化钇等以及上述氧化 物中若干种的混合物 ${ }^{[6]}$ 。粉体原料的化学成分对单 片层形成过程的影响主要体现在该物质固有的密 度、比热、热传导能力以及热膨胀水平等方面。例 如同样使用大气等离子体喷涂技术, 相比于氧化钇 稳定的氧化锆(Yttria-stabilized Zirconia, YSZ), 以氧 化铝为原料制备的单片层通常微裂纹更稀疏。仅以 粉体成分为变量开展研究的内容未见报道, 与之有 关的研究通常将影响因素分解为粉体颗粒尺寸、粉 体形貌及其他因素。

\section{2 粉体颗粒尺寸}

喷涂过程使用的粉体具有特定的尺寸分布。用 以制备热障涂层的一种常见的商用原料是 Oerlikon Metco(原 Sulzer Metco)公司牌号为 204NS 的 YSZ 粉体, 其粒径主要集中在 $11 \sim 125 \mu \mathrm{m}$ 。就单个粉体
颗粒而言，我们将粉体颗粒尺寸对喷涂工艺的影响 归结为三个相互关联的方面，即：

(1)影响粉体进入等离子体焰流的能力。以具有 代表性的 Oerlikon Metco 公司 F4MB 喷枪制备 YSZ 涂层工艺为例, 粉体垂直进入等离子体焰流(图 1)。 在常规送粉载气压力条件下，尺寸过大的颗粒会穿过 温度最高的等离子体焰流中心区域, 无法被有效加热; 粒径较小 $(<10 \mu \mathrm{m})$ 的颗粒由于动量小, 不能穿过高速 等离子体焰流与环境气氛之间的界面，仅能“漂浮”在 火焰外表，同样难以被有效加热。虽然增大送粉载气 的压力可以增大粉体颗粒垂直方向的速率，但过大的 压力将对等离子体焰流造成干扰, 因而粒径极小 $(<5 \mu \mathrm{m})$ 的粉末是难以直接使用的 ${ }^{[6]}$ 。

(2)影响粉体在等离子体焰流中温度和速率的提 升。一些研究者 ${ }^{[8-11]}$ 以模拟方式分析了原料颗粒、载气 流量、功率等参数对熔滴温度和速率的影响。颗粒能 够进入到等离子体焰流的程度不同，而等离子体焰流 自身的温度和流速也不均匀, 导致熔滴最终的温度和 速率结果较为复杂。Elsebaei 等 ${ }^{[12]}$ 以实验方式比较了偏 离喷枪中心不同距离的位置所观测到的熔滴在与基 体碰撞前的直径、速率和表面温度, 结果列于表 1 。 从表 1 可以看出, 在给定的实验条件下, 等离子体焰 流中心区域对颗粒的加热和加速能力是比较好的; 粒 径较小的颗粒无法进入等离子体焰流中心, 最终获得 的温度和飞行速率较小; 而粒径较大的颗粒穿过了等 离子体焰流中心, 最终获得的温度和飞行速率更小。

(3) 影响熔滴在碰撞祄底后的冷却固化与铺展 的竞争关系。当熔滴接触祄底, 一方面热量快速向 祄底传递, 熔滴发生高速的定向冷却; 另一方面, 撞击改变了熔滴内部液态物质的运动方向, 使其在 衬底平面上背离熔滴中心向外铺展。单片层的扁平 比以及是否溅射等特征都可以归结为这两种机制的 综合作用结果。

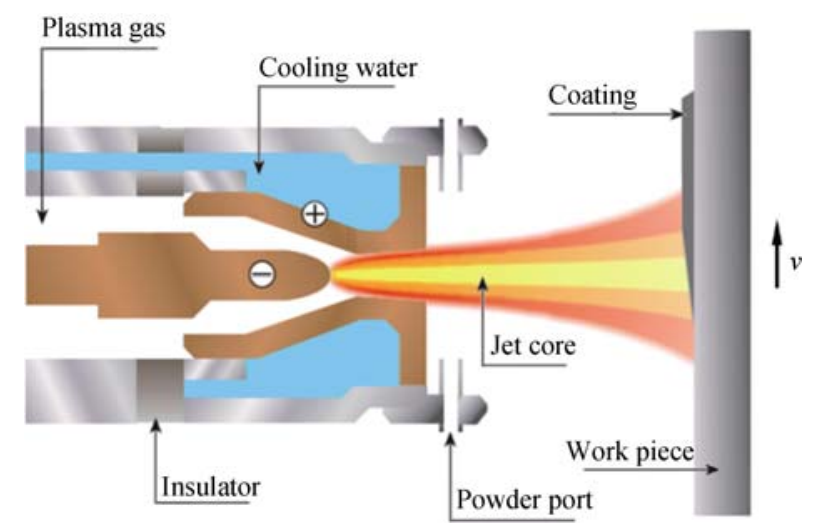

图 1 常见的等离子体喷枪结构示意图

Fig. 1 Schematic drawing of typical plasma torch 
表 1 不同轴向位置处颗粒的直径、速率及温度的平均值 ${ }^{[12]}$

Table 1 Average values of the particle size, velocity and temperature at different radial positions from the plasma jet center line ${ }^{[12]}$

\begin{tabular}{cccc}
\hline $\begin{array}{c}\text { Radial po- } \\
\text { sition } / \mathrm{mm}\end{array}$ & $\begin{array}{c}\text { Average parti- } \\
\text { cle diameter } \\
/ \mu \mathrm{m}\end{array}$ & $\begin{array}{c}\text { Average particle } \\
\text { velocity } /\left(\mathrm{m} \cdot \mathrm{s}^{-1}\right)\end{array}$ & $\begin{array}{c}\text { Average particle } \\
\text { temperature } / \mathrm{K}\end{array}$ \\
\hline+50 & 25.7 & 236 & 3310 \\
+40 & 26.3 & 247 & 3342 \\
+30 & 27.8 & 254 & 3392 \\
+20 & 30.3 & 248 & 3430 \\
+10 & 32.3 & 233 & 3420 \\
0 & 36.5 & 228 & 3400 \\
-10 & 37.0 & 215 & 3340 \\
-20 & 38.2 & 209 & 3310 \\
-30 & 42.3 & 199 & 3294 \\
-40 & 44.3 & 191 & 3270 \\
-50 & 45.6 & 188 & 3250 \\
\hline
\end{tabular}

\section{3 粉体形貌}

仅以商品化的粉体原料为例, Oerlikon Metco 就 推出了多种形貌的产品 ${ }^{[13]}$, 如图 2 所示。粉体形貌 与其在送粉管内运动过程中所受的阻力关系密切, 还会直接影响载气和等离子体焰流对粉体的作用。 此外, 粉体形貌的差异还影响了熔滴与祄底的碰撞 过程。Kumar 等 ${ }^{[14]}$ 模拟了具有相同外径和温度的空 心熔滴与致密熔滴形成单片层的过程，在该实验的 设定条件下，空心熔滴撞击祄底后会有一部分物质 反向溅离，且固化需要的时间更长，但形成的单片 层却反而更厚。

\section{4 其他}

熔滴与祄底碰撞前的温度和速度对形成单片层 的作用极为重要。这两个因素中还有一些问题应该 特别注意: 速度的方向和熔滴温度的不均匀性。
根据等离子体焰流与祄底表面的位置关系，颗 粒的飞行角度可以分为垂直、偏离角度较小以及偏 离角度较大三种, 其中后两种情况一般以 $45^{\circ}$ 为分 界。实验研究结果显示, 在偏离角度较大的情况下, 单片层的形貌会出现显著变化，所制备涂层的气孔 率和表面粗糙度增大, 机械性能降低 ${ }^{[15-16]}$ 。

通常粉体颗粒在等离子体焰流中停留的时间不 足以使其达到内核和外壳温度均匀的理想状态，特 别是对于比较致密的陶瓷粉体, 往往外壳已经变为 液态而内核仍保持固态。图 3 是 Alavi 等 ${ }^{[17]}$ 基于这 类两相模型获得的模拟对比结果。Zhu 等 ${ }^{[18]}$ 使用了 温度分布更复杂的模型, 进一步分析了内核尺寸对 所形成单片层过程的影响。在研究工作中，应该依 据所用喷枪的功率水平以及粉体的尺寸和形貌等, 合理地选择熔滴模型。

还有研究者从更宏观的角度, 以雷诺数 ${ }^{[19-20]}$ 、

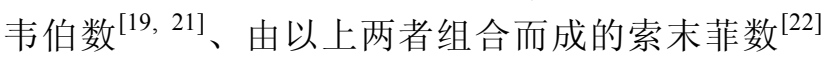
或熔融指数 ${ }^{[23-24]}$ 等无量纲数来描述熔滴的运动状 态。虽各有不同, 但都与本文以运动速度及温度为 核心参数的观点无本质冲突，因为其中涉及的质 量、密度、粘度、表面张力等参数对于特定熔滴体 系是几乎不变或与温度和速度密切相关的。

\section{2 喷涂的客体：衬底}

述及客体因素对单片层形成过程的影响之前, 需 先明确两个被广泛使用的概念: 转变温度(transition temperature, $T_{\mathrm{t}}$ ) 和冷却速率(cooling rate)。

Fukumoto 等 ${ }^{[25]}$ 在抛光表面用金属镍的熔滴制 备单片层, 发现当衬底的温度提高至某一范围很窄 的区间以上时，单片层从溅射状转变成了规则的圆 盘状。这一温度区间被简化为转化率 $50 \%$ 所对应的 温度, 称为转变温度。这种转变现象与通常理解中

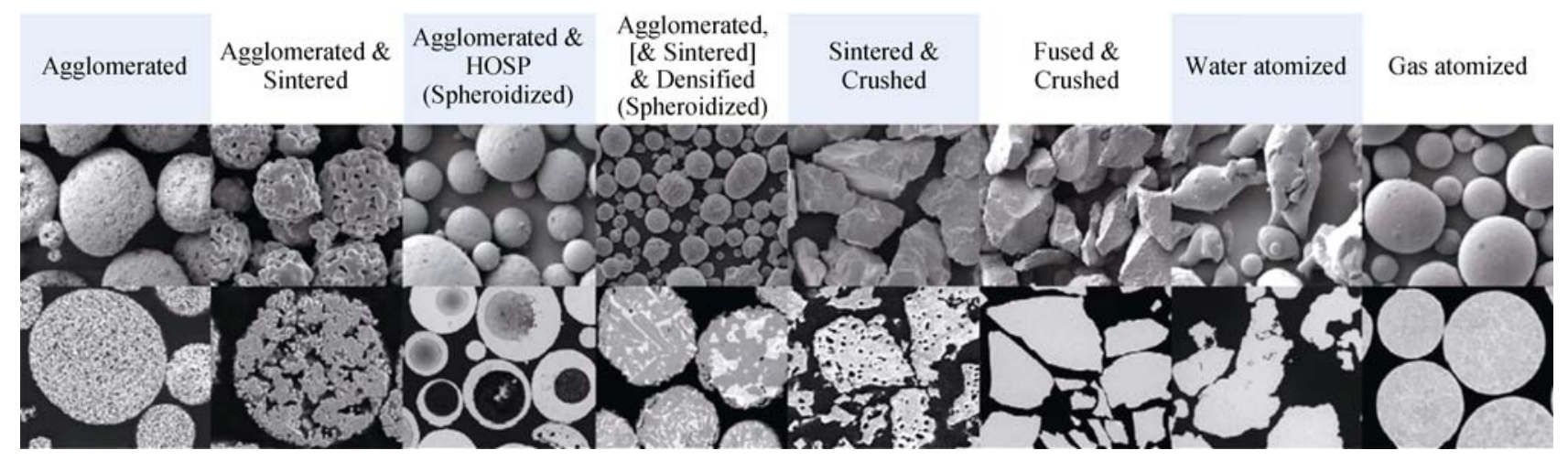

图 2 市售 Oerlikon Metco 公司部分陶瓷粉体原料的外观和内部特征 ${ }^{[13]}$

Fig. 2 Shape and interior morphologies of the ceramic powder provided by Oerlikon Metco ${ }^{[13]}$ 

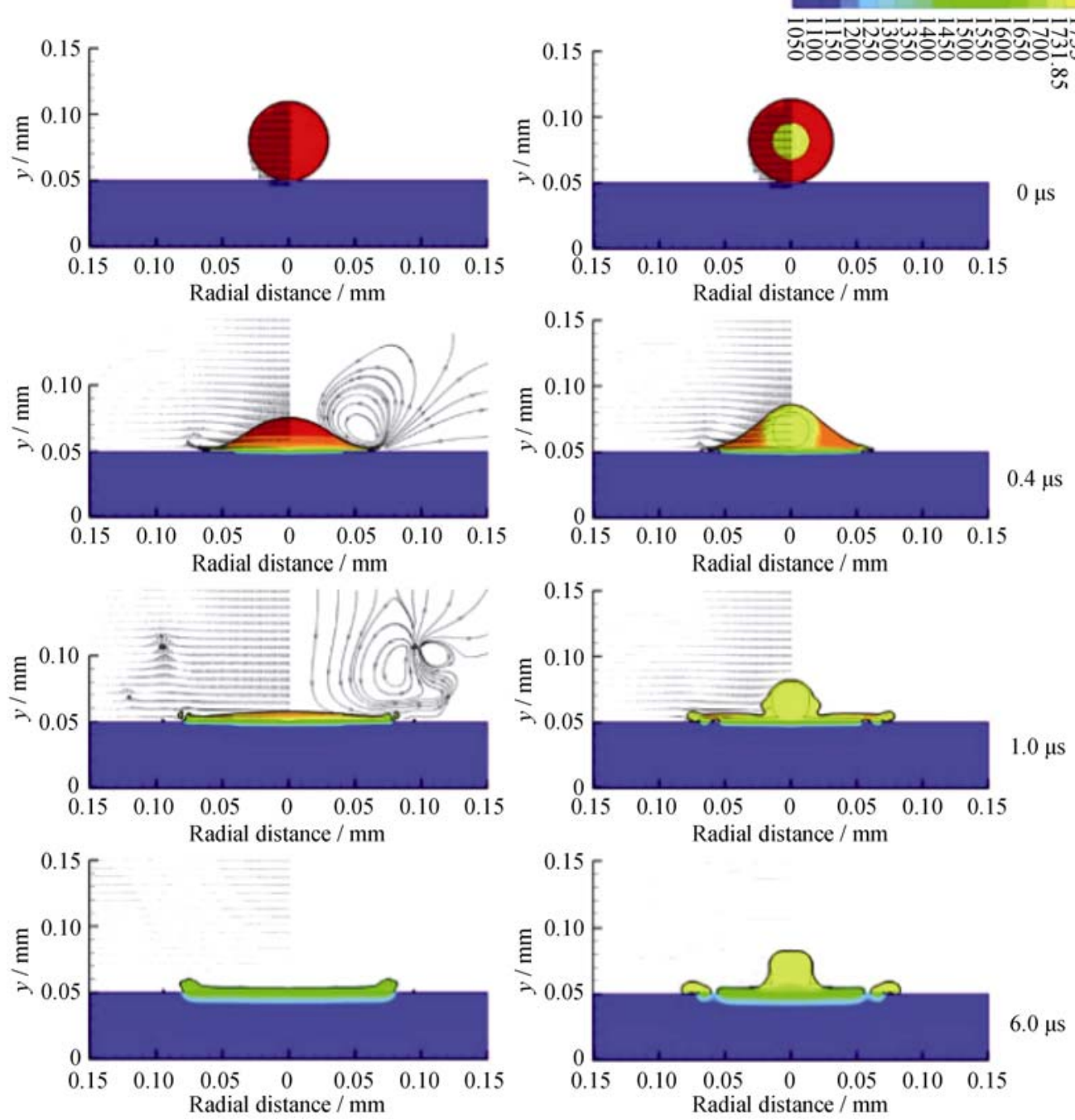

图 3 模拟完全熔融与半熔融状态镍金属颗粒形成单片层过程 ${ }^{[17]}$

Fig. 3 Computer-generated images of the normal impact of a molten and a semi-molten nickel droplet ${ }^{[17]}$

的情况是相悖的。单片层的溅射是形成过程中铺展 优于冷却成为主要因素的结果。基体温度升高使其 与熔滴底部的温度梯度变小, 造成更低的热传递速 率, 而这显然利于铺展而非冷却。

相比于转变温度, 冷却速率与单片层形貌差异 的内在形成机制之间的关联更为直接。上世纪 90 年代, Moreau 等 ${ }^{[26]}$ 使用响应时间为 $100 \mathrm{~ns}$ 的高温计 测量了钼熔滴在玻璃祄底表面形成单片层过程中的 冷却时间，该实验的原理如图 4 所示。在此基础上， Fauchais 等 ${ }^{[27]}$ 测量了 $8 \mathrm{YSZ}$ 粉体颗粒在不同粗糙度 的 304L 不锈钢和 $8 \mathrm{YSZ}$ 表面铺展的平均冷却速率。 除了定量测量，文献[28]还报道过一种利用单片层 中柱状晶的直径变化来比较冷却速率相对大小的方 法, 其原理是同种物质晶粒尺寸的平方与固化速率 的乘积是一个常数。

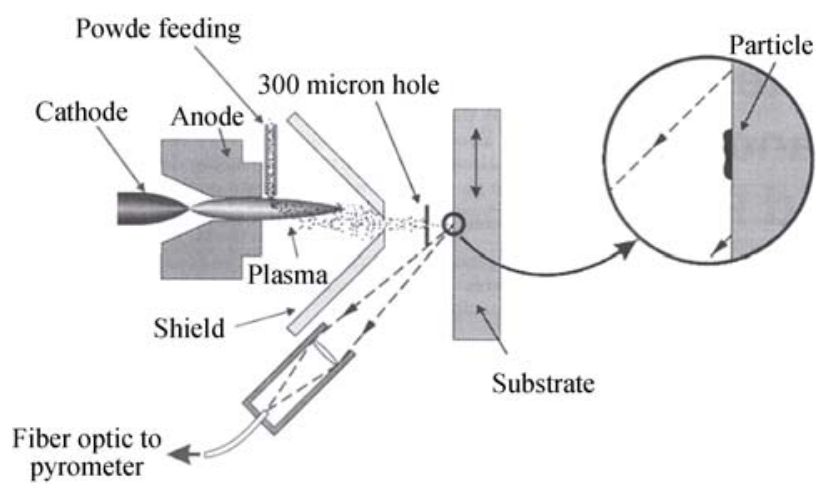

图 4 测量钿液滴冷却时间的设备及其原理 ${ }^{[26]}$

Fig. 4 Schematic of the experimental setup and typical signals collected during an impact of a molybdenum droplet on a smooth glass substrate $\mathrm{e}^{[26]}$

此外, 一些研究者 ${ }^{[29-32]}$ 还经常使用“接触热阻 (thermal contact resistance)”这一参数来描述熔滴向祄 底的热传递能力, 其值通常为 $10^{-6} \sim 10^{-8} \mathrm{~m}^{2} \cdot \mathrm{K} / \mathrm{W}^{[29]}$ 。本 
文倾向于将之视为各影响因素的综合结果, 对于原 料确定的特定体系而言, 所谓影响因素通常包括以 下几个方面:

\section{1 衬底种类}

祄底的种类直接决定了祄底的导热能力以及祄 底与熔滴之间的润湿性。定性来看, 衬底的导热能 力越强、与熔滴的润湿性越好, 熔滴的冷却速率越 快、越不容易发生溅射。具体地, 有关基体导热能 力对单片层形成影响的一个比较有影响力的实验是: Fukumoto 等 ${ }^{[25]}$ 以粒径约为 $45 \mu \mathrm{m}$ 的金属镍颗粒为 原料, 以热喷涂的方法使其铺展在不同种类的祄底 上。为了减小润湿性差异导致的误差, 各基体表面 镀覆了一层较薄的金膜。该实验结果显示, 基体材 料的热导率越高, 转变温度越高。Fukumoto 等 ${ }^{[25]}$ 还发现, 与祄底材料的润湿性越好, 镍单片层的转 变温度越低。然而, 关于润湿性对单片层形貌的影 响, 也有研究者对上述结论持有疑议。Fukumoto 等 ${ }^{[33]}$ 也曾指出, 熔滴铺展过程的推进接触角(advancing contact)为疏 “水”类型, 加之氧化导致的表面粗糙, 理论 上不利于圆盘状单片层的形成。此外, 考虑到热喷 涂过程中熔滴形成单片层的时间是极短的 $\left(<10 \mu \mathrm{s}^{[7]}\right)$, 本文认为狭义的润湿性观点不是影响单片层形成的 决定因素。将实际接触面积与表观接触面积之比也宽 泛地解释为“润湿性”, 虽然没有谬误, 但不够准确。

\section{2 衬底温度}

衬底温度是等离子体喷涂工艺中一个重要且易 于调控的工艺参数。有关祄底温度与涂层性能之间 的关系研究在文献中所占比例较大。但是, 多数文 献在探讨这一问题时都将“温度”与“预热”赋予了相
同的含义, 把祄底温度的影响归结为预热导致的祄 底表面氧化的影响。

前文已经介绍了转变温度的概念。Fukumoto 等 ${ }^{[34]}$ 在对金属铜熔滴的铺展行为的研究中分析了祄底温 度影响单片层形貌的作用机理，提出了图 5 所示的 模型。该理论认为, 对于温度低于转变温度的祄底, 熔滴底部在与之碰撞初期就发生了局部的迅速固化, 使得可以提供能量传输的通道非常有限; 熔滴上部 由于温度降低缓慢, 铺展占据优势, 因而具有更大 的溅射倾向。反之, 如果基体的温度较高, 由于与熔 滴接触部分的温度梯度较小，反而形成了较好的接 触, 虽然初期冷却较慢, 但面积更大的导热通道实 际上提高了熔滴上部液态物质的冷却速率，使整个 单片层的冷却占据优势, 不易发生溅射。Fauchais ${ }^{[7]}$ 指出, 前一种情况的接触面积占表观面积的比例仅 为 $10 \% \sim 20 \%$, 而后一种情况下这一数值可以达到 $80 \%$ 以上。这种“初期激冷”的假说很好地解释了转 变温度的成因, 且与实验中观察到的沉积在玻璃表 面的单片层的底部形貌特征一致。但是悬殊的平均 接触面积仅靠温度梯度导致的冷却速率来解释仍不 充分, 例如这一理论也不能很好地解释转变温度区 间为什么非常狭窄。

\section{3 衬底表面吸附}

不少研究者 ${ }^{[35-37]}$ 都关注了祄底表面吸附物对单 片层形貌的影响。其中 $\mathrm{Li}$ 等 ${ }^{[36]}$ 将沸点在 $144 \sim 290^{\circ} \mathrm{C}$ 的 三种化合物涂覆在光滑的不锈钢基体表面，观察不 同预热温度条件下单片层的形貌特征：当预热温度 低于预涂覆化合物沸点时，获得的单片层呈现显 著的溅射状; 而预热温度高于化合物沸点 $50^{\circ} \mathrm{C}$

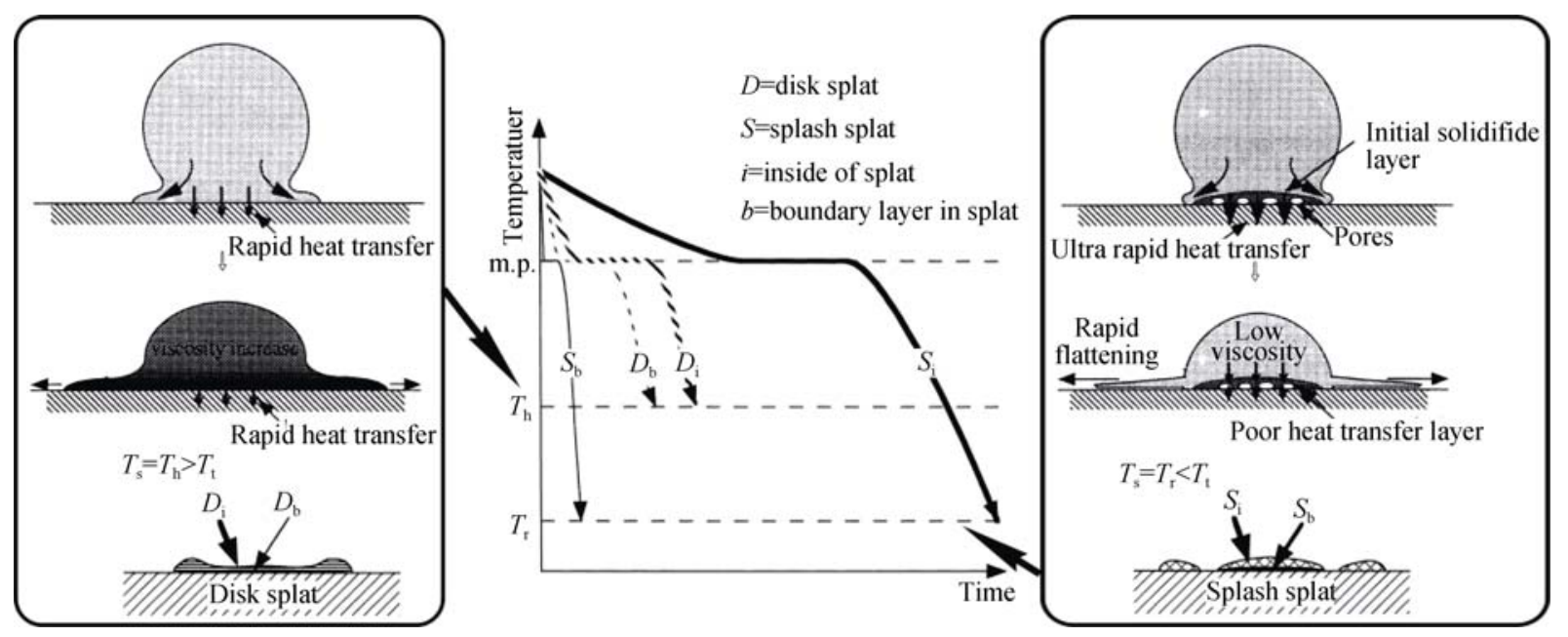

图 5 碰撞初期的激冷导致单片层呈溅射状的机理 ${ }^{[34]}$

Fig. 5 Comparison of cooling and solidification processes inside the splat deposited onto substrates at a temperature above (left) and below (right) transition temperature ${ }^{[34]}$ 
以上, 获得的单片层呈现圆盘状。这表明有机物 受热气化可以显著改变熔滴铺展前沿的流动方向, 进而引起溅射。这一观点与狭窄的转变温度区间 能较好对应。

然而, 在实际研究或生产中, 喷涂之前一般都 会以酒精或丙酮清洗工件。基体表面存有大量有机 污染物的可能性较小。Tran 等 ${ }^{[38]}$ 将上述研究中的 有机物换成了基体表面在空气中形成的羟基和吸附 水, 并具体指出其中羟基的作用更为核心。

\section{4 衬底表面氧化}

在预热过程中，金属基体的表面会形成明显的 氧化层(事实上, 没有加热的金属表面仍有氧化层 存在, 只是厚度非常小)。氧化层对单片层形成过 程的影响可以归为如下几类:(1)相当于改变了基体 材质, 影响基体的表面吸附状态以及熔滴与基体之 间的“润湿性”。(2)氧化物是热的不良导体, 阻碍了熔 滴向基体的热量传递。McDonald 等 ${ }^{[39]}$ 根据 $R_{\text {oxide }}=$ $t / k$ (其中 $t$ 为氧化层的厚度, $k$ 为氧化物的热导率)估算出 厚度数百纳米的氧化层的热阻约为 $3.4 \times 10^{-8} \mathrm{~m}^{2} \cdot \mathrm{K} / \mathrm{W}$, 而该研究小组测得的单片层形成过程中熔滴与基体 总的热阻约为 $10^{-6} \sim 10^{-7} \mathrm{~m}^{2} \cdot \mathrm{K} / \mathrm{W}$, 进而认为氧化层热 阻可以忽略。但该计算中没有考虑氧化层与金属之 间的界面热阻，也没有考虑氧化层本身的不致密性， 数据或偏于保守。(3)厚度较大的氧化层极大地改 变了基体的表面形貌, 这一影响在众多研究者的论 文中都有提及, 是预热改变单片层形貌最为重要的 机理。

\section{5 衬底表面粗䊁度}

Parizi 等 ${ }^{[40]}$ 将基体的表面形貌抽象为具有周期 性的方块突起, 仿真计算研究了祄底表面周期性形 貌特征对熔滴铺展的影响, 并以刻蚀的硅片为基体 进行了实验验证(图 6)。该研究发现, 熔滴在铺展过 程中并不能完全填满粗䊁祄底表面的孔/槽, 尤其是

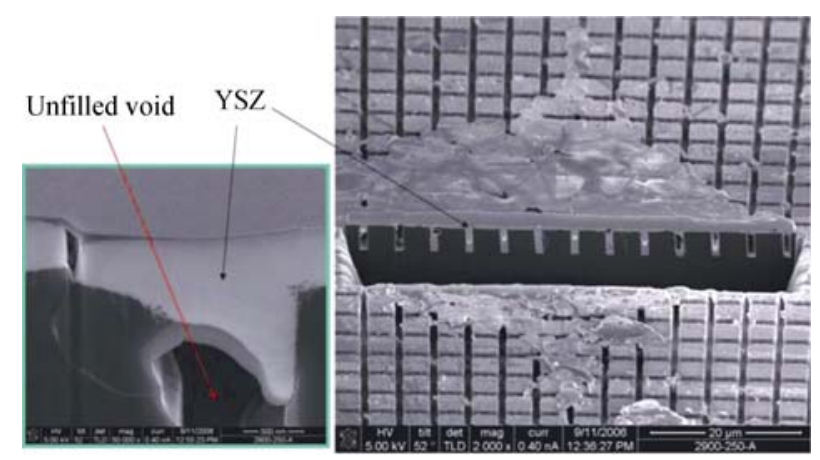

图 6 在经过刻蚀处理的硅表面铺展的 YSZ 熔滴形貌 ${ }^{[40]}$

Fig. 6 SEM images of YSZ splats on patterned silicon surface $^{[40]}$
在远离碰撞中心的位置。Shinoda 等 $^{[41]}$ 也报道过类 似的模拟工作，但使用的是扁圆柱形微坑规则排列 的表面模型。

相比于模拟计算，以喷砂或预氧化等手段制 备具有不同形貌特征表面的实验研究更为常见。 喷砂使用的刚玉砂直径通常大于 $0.71 \mathrm{~mm}$, 而大气 等离子体喷涂制备的 YSZ 单片层的直径一般约为 $100 \mu \mathrm{m}$ 。以单片层的尺度考量, 经过喷砂处理的 表面几乎是平坦光滑的。喷砂往往与涂层整体(而 不是单个的单片层)性能相联系, 如 Khan 等 ${ }^{[42]}$ 关 注的喷砂工艺对涂层性能的影响。也有研究关注 了喷砂表面单片层的形貌，如 Christoulis 等 ${ }^{[43]}$ 统计 了抛光表面(平均表面粗楉度 $R_{\mathrm{a}}=0.02 \mu \mathrm{m}$ ) 与喷砂表 面 $\left(R_{\mathrm{a}}=1.33 \mu \mathrm{m}\right)$ 沉积的金属单片层中的晶粒尺寸 并计算了形成过程中的过冷度，发现相同基体温 度条件下喷砂表面单片层的晶粒尺寸比抛光表面 单片层的略大。

经预氧化的表面, 其特征尺寸约为 $0.1 \sim 10 \mu \mathrm{m}^{[44]}$ 。 相比于喷砂，这种前处理方法对单片层形成的影响更 为显著, 成为本领域研究的热点之一。Fukumoto ${ }^{[33]}$ 、 Syed $^{[45]}$ 以及 Salimijazi ${ }^{[46]}$ 等都注意到了预热使得基 体表面形貌发生改变的现象。McDonald 等 ${ }^{\left[{ }^{[39}\right.}$ 在原 子力显微镜下观察了 $304 \mathrm{~L}$ 不锈钢经不同温度预氧 化 $30 \mathrm{~min}$ 后的表面形貌，并比较了常温条件下上述 表面获得的金属镍单片层的最终形貌和形成过程中 的冷却速率(图 7)。该实验显示, 因氧化而变得粗 粘的表面使得熔滴与祄底之间的接触面积增大, 因 此增大了单片层形成过程中的冷却速率。这一研究 结果与 Shinoda 等 ${ }^{[41]}$ 和 Christoulis 等 ${ }^{[43]}$ 的观点并不 一致, 但与其他研究者报道的实验现象及分析基本 相符。

进一步地, 如果祄底表面与熔滴的接触面积可 用以解释单片层形成过程中的冷却速率, 那么宏观 上哪些参数能有效与之对应呢? $\mathrm{Li}$ 等 ${ }^{[44]}$ 尝试以周 期和振幅相差较大的正弦函数之和来描述祄底表面 的形貌特征, 并指出通常使用的 $R_{\mathrm{a}}$ (平均表面粗粘 度)的定义使该参数对祄底表面特征尺寸较大的起 伏(即周期和振幅数值较大的项)更为敏感, 并不能 有效表征特征尺寸与单片层厚度相近的微小突起， 而这些微小的形貌特征对单片层形成过程的影响才 是最为重要的。Fukumoto 等 ${ }^{[33]}$ 也报道 $R_{\mathrm{a}}$ 大小相似 的祄底表面的微观形貌可能有很大的差异; 对应地, 获得的单片层形貌也相差很大。经比较, Fukumoto 等 ${ }^{[33}$ 认为 $R_{\mathrm{sk}}$ (轮廓的偏斜度)能更有效地对应单片 层的形貌特征。然而这一观点在 McDonald 等 ${ }^{[39]}$ 的 

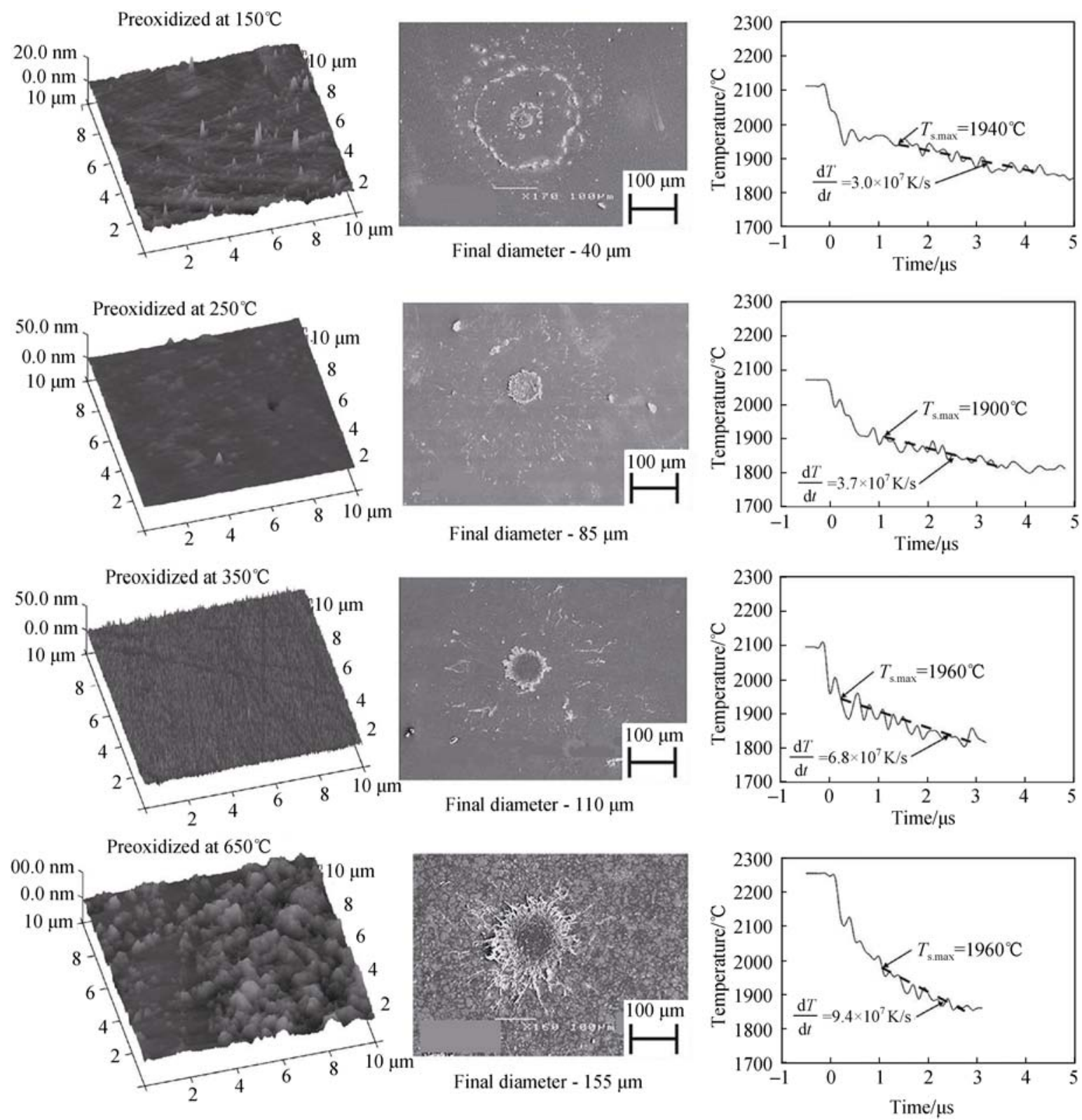

图 7 经不同温度预氧化处理后的不锈钢表面形貌、所沉积镍单片层的形貌以及单片层形成过程中冷却速率的对比 ${ }^{[39]}$

Fig. 7 Surface topologies of stainless steel surfaces either non-oxidized or preoxidized at different temperatures, images of nickel splats after solidification and cooling curves of splats ${ }^{[39]}$

研究中遭到了否定。 $\mathrm{Li}$ 等 ${ }^{[44}$ 认为以上报道的矛盾或 许是因为通常使用的表面粗楉度仪的探针尺寸较大, 所测结果不能准确代表祄底表面周期和振幅较小的 突起。目前研究者在衬底表面的表征这一技术细节 上尚未取得一致的观点。

\section{3 环境因素及其他}

喷涂所处空间的环境，特别是气氛和压力，对 等离子体焰流及熔滴形成单片层的过程影响显著。 尤其是近年来快速发展的低压、大功率等离子体喷 涂技术，环境因素的影响不言而喻。本文主要是基
于大气等离子体喷涂工艺，加之篇幅限制，环境因 素的影响将不做详细介绍。与本文着力探讨的单片 层形成过程中的相关机理有较大关联的是 Fukumoto 等 ${ }^{[28]}$ 以金属铜熔滴沉积单片层的一组对比研究, 实 验中基体为抛光态 AISI304 不锈钢, 环境气氛为氮气, 压力分别被设置为 $107 \mathrm{kPa}$ 和 $1 \mathrm{kPa}$ 。该实验发现, 在低压环境下，即使不加热基体，沉积的单片层也 呈圆盘状; 对比在不锈钢基体表面沉积的单片层, 低压组在不加热祄底的条件下与高压组在祄底被加 热到转变温度以上的条件下所沉积的单片层的晶粒 尺寸是相当的(图 8)。

此外, 在更宽泛的热喷涂研究中, 还有一些在 


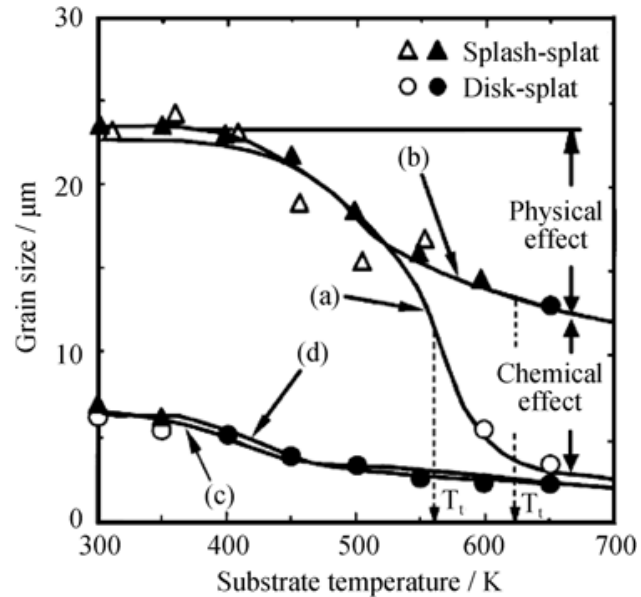

图 8 温度、气压和基体表面成分对铜单片层外形及晶粒尺 寸的影响 ${ }^{[28]}$

Fig. 8 Grain size in a cross-section of the splat obtained in different ambient pressures ${ }^{[28]}$

(a) on stainless steel in atmospheric pressure; (b) on gold-coated stainless steel in atmospheric pressure; (c) on stainless steel under low pressure; and (d) on gold-coated stainless steel under low pressure

特定体系和条件下才可能出现的现象, 如 $\mathrm{Li}$ 等 ${ }^{[47]}$ 在大功率 $(>80 \mathrm{~kW})$ 喷涂条件下, 沉积在不锈钢表面 的钼金属熔滴会使下方的基体局部融化，进而引起 熔滴在铺展过程中液态物质前进方向的改变, 使单 片层呈现溅射状。Brossard 等 ${ }^{[48]}$ 在研究铝表面 Ni$\mathrm{Cr}$ 熔滴铺展时发现界面处形成了中间相, 这种现象 使涂层在热循环过程中的热失配应力减小，与基体 的结合强度也得到大幅提高。

\section{4 总结与展望}

单片层是热喷涂制备涂层的结构基元, 其特征 与涂层的宏观性能密切相关。大气等离子体喷涂工 艺可控制备涂层的难点在于工艺中需要控制的因素 很多，且往往相互影响；熔滴高温、高速和理化状 态分布广等特性给实时观测和工艺的精确调控也带 来了很大的挑战。本文综述了一些文献中报道的单 片层形成过程中的各类影响因素。需要指出, 文中列 举的各种因素并非严格并列关系, 很多要素之间存 在紧密的内在关联，这也体现了对大气等离子体喷 涂工艺研究和涂层可控制备的探索过程的复杂性。

大气等离子体喷涂工艺中单片层的形成过程主 要是受到熔滴冷却能力的控制。熔滴冷却速率快, 则液态物质流动性降低快, 倾向于形成圆盘状的单 片层; 反之则具有较强的溅射趋势。单片层外形的 改变带来的一个与涂层性能密切相关的差异是：圆 盘状单片层和祄底之间的结合更牢固, 而溅射状单
片层和祄底的结合强度相对较低 ${ }^{[33]}$ 。

众多学者在 APS 单片层研究领域已经取得了很 多有益的研究成果。同时还需指出, 这些研究中往 往以尺寸较大的金属熔滴和表面光滑的基体为研究 对象。这类研究方法的优势在于能便捷地监测熔滴 速度和温度，获得的单片层也更易于观察。此外， 还能方便地将获得的单片层从衬底表面剥离以观察 单液滴底部与祄底界面处的特征。但同时也导致这 类研究与实际应用条件下高温、高速的陶瓷熔滴在 粗糙的表面铺展固化形成单片层的情形有极大的差 异。尽管研究者有意在实验中设计了与真实条件相 近的流体力学参数作为实验条件, 但获得的数据仍 可能与实际结果出现不可预判的偏差。现有文献中 出现的与其他研究者所报道现象和规律相悖的情形 很有可能与上述类比过程中的差异有关。

更可靠的原位温度、速度监测, 特别是能追踪 单个熔滴的温度和速度测量无疑是研究工艺参数对 单片层特点影响的最直接有效的方法。然而大气等 离子体喷涂工艺中高温、高速的特点使得这种观察 异常困难。对于具有熔滴尺寸更小、喷涂功率更大 以及低压喷涂环境等特征的新型喷涂技术，这样的 观测变得更加困难。对于未来有关单片层的研究, 较为实际并可靠的方法应尽可能选用实际应用的喷 涂材料和设备, 对粉体原料进行预加工使之具有极 窄的粉体粒径分布，充分考虑已有研究中指出的干 扰因素, 以大量单片层观测结果来考察目标变量的 影响及其规律; 而模拟研究过程中所设的边界条件 和影响因素也应该建立在符合上述条件的观察结果 之上。此外, 将单片层的形貌特点数据化并使之与 涂层整体性能间建立(半)定量关系也是未来发展的 一个重要方向。

\section{参考文献:}

[1] 徐滨士, 李长久, 刘世参, 等. 表面工程与热喷涂技术及其发展. 中国表面工程, 1998, 38(1): 3-9.

[2] 徐滨士, 马世宁, 时小军, 等. 中国表面工程的发展. 中国机械 工程, 1996, 7(5): 3-5.

[3] 曹学强. 热障涂层新材料和新结构. 北京: 科学出版社, 2016.

[4] 黄小鸥, 吴朝军. 适应产业结构调整, 开发新型涂层产品. 第十 七届国际热喷涂研讨会(ITSS'2014)暨第十八届全国热喷涂年会 (CNTSC'2014), 成都\&自贡, 中国, 2014: 1-5.

[5] 中国表面工程协会热喷涂专业委员会. 中国热喷涂年鉴: 2015 年版. 北京: 科学技术文献出版社, 2016 .

[6] FAUCHAIS P, HEBERLEIN J, BOULOS M. Thermal Spray Fundamentals: from Powder to Part. New York: Springer Sci- 
ence+Business Media, 2014 .

[7] FAUCHAIS P. Understanding plasma spraying. J. Phys. D Appl. Phys., 2004, 37(9): 86-108.

[8] VARDELlE M, VARDELLE A, DUSSOUBS B, et al. Influence of injector geometry on particle trajectories: analysis of particle dynamics in the injector and plasma jet. Thermal Spray, Vols 1 and 2, 1998: 887-894.

[9] LI H P, CHEN X. Three-dimensional simulation of a plasma jet with transverse particle and carrier gas injection. Thin Solid Films, 2001, 390(1/2): 175-180.

[10] XIONG H B, ZHENG L L, SAMPATH S, et al. Three-dimensional simulation of plasma spray: effects of carrier gas flow and particle injection on plasma jet and entrained particle behavior. Int. J. Heat Mass Tran., 2004, 47(24): 5189-5200.

[11] YUGESWARAN S, KOBAYASHI A, SELVAN B, et al. In-flight behavior of lanthanum zirconate $\left(\mathrm{La}_{2} \mathrm{Zr}_{2} \mathrm{O}_{7}\right)$ particles in gas tunnel type plasma jet and its coating properties. Vacuum, 2013, 88: 139-143.

[12] ElSEBAEI A, HEBERLEIN J, ELSHAER M, et al. Comparison of in-flight particle properties, splat formation, and coating microstructure for regular and nano-ysz powders. J. Therm Spray Techn., 2010, 19(1/2): 2-10.

[13] OERLIKON METCO. Thermal spray materials guide. https:// www.oerlikon.com/metco/en/products-services/coating-materials/c oating-materials-thermal-spray/, April 2015.

[14] KUMAR A, GU S, TABBARA H, et al. Study of impingement of hollow $\mathrm{ZrO}_{2}$ droplets onto a substrate. Surf. Coat. Tech., 2013, 220: 164-169.

[15] KANOUFF M P, NEISER R A, ROEMER T J. Surface roughness of thermal spray coatings made with off-normal spray angles. $J$. Therm. Spray Techn., 1998, 7(2): 219-228.

[16] LEIGH S H, BERNDT C C. Evaluation of off-angle thermal spray. Surf. Coat. Tech., 1997, 89(3): 213-224.

[17] ALAVI S, PASSANDIDEH-FARD M, MOSTAGHIMI J. Simulation of semi-molten particle impacts including heat transfer and phase change. J. Therm. Spray Techn., 2012, 21(6): 1278-1293.

[18] ZHU Z H, KAMNIS S, GU S. Numerical study of molten and semi-molten ceramic impingement by using coupled Eulerian and Lagrangian method. Acta Mater., 2015, 90: 77-87.

[19] GOUTIER S, VARDELLE M, FAUCHAIS P. Comparison between metallic and ceramic splats: influence of viscosity and kinetic energy on the particle flattening. Surf. Coat. Tech., 2013, 235: $657-668$.

[20] CHEN D, WANG Y, BAI Y, et al. Effect of reynolds number of molten particle on splat formation in plasma spraying. J. Inorg. Mater., 2015, 30(1): 65-70.

[21] GOUTIER S, VARDELLE M, LABBE J C, et al. Flattening and cooling of millimeter- and micrometer-sized alumina drops. $J$. Therm. Spray Techn., 2011, 20(1/2): 59-67.

[22] ESCURE C, VARDELLE M, FAUCHAIS P. Experimental and theoretical study of the impact of alumina droplets on cold and hot substrates. Plasma Chem. Plasma P., 2003, 23(2): 185-221.

[23] LI L, VAIDYA A, SAMPATH S, et al. Particle characterization and splat formation of plasma sprayed zirconia. J. Therm. Spray Techn., 2006, 15(1): 97-105.

[24] WANG Y Z, HUA J J, LIU Z W, et al. Melting index characterization and thermal conductivity model of plasma sprayed YSZ coatings. J. Eur. Ceram. Soc., 2012, 32(14): 3701-3707.

[25] FUKUMOTO M, HUANG Y. Flattening mechanism in thermal sprayed nickel particle impinging on flat substrate surface. $J$. Therm. Spray Techn., 1999, 8(3): 427-432.

[26] MOREAU C, GOUGEON P, LAMONTAGNE M. Influence of substrate preparation on the flattening and cooling of plasmasprayed particles. J. Therm. Spray Techn., 1995, 4(1): 25-33.

[27] FAUCHAIS P, VARDELLE M, VARDELlE A, et al. Parameters controlling the generation and properties of plasma sprayed zirconia coatings. Plasma Chem. Plasma P., 1996, 16(1): 99-125.

[28] FUKUMOTO M, NISHIOKA E, MATSUBARA T. Effect of interface wetting on flattening of freely fallen metal droplet onto flat substrate surface. J. Therm. Spray Techn., 2002, 11(1): 69-74.

[29] FAUCHAIS P, FUKUMOTO M, VARDELLE A, et al. Knowledge concerning splat formation: an invited review. J. Therm. Spray Techn., 2004, 13(3): 337-360.

[30] MCDONALD A, MOREAU C, CHANDRA S. Thermal contact resistance between plasma-sprayed particles and flat surfaces. Int. J. Heat Mass Tran., 2007, 50(9/10): 1737-1749.

[31] TRAN A T T, HYLAND M M. The role of substrate surface chemistry on splat formation during plasma spray deposition by experiments and simulations. J. Therm. Spray Techn., 2010, 19(1/2): $11-23$.

[32] ZHENG Y Z, LI Q, ZHENG Z H,et al. Modeling the impact, flattening and solidification of a molten droplet on a solid substrate during plasma spraying. Appl. Surf. Sci., 2014, 317: 526-533.

[33] FUKUMOTO M, OHGITANI D, YASUI T. Effect of substrate surface change on flattening behaviour of thermal sprayed particles. Mater Trans., 2004, 45(6): 1869-1873.

[34] FUKUMOTO M, NISHIOKA E, MATSUBARA T. Flattening and solidification behavior of a metal droplet on a flat substrate surface held at various temperatures. Surf. Coat. Tech., 1999, 120: 131-137.

[35] JIANG X Y, WAN Y P, HERMAN H, et al. Role of condensates and adsorbates on substrate surface on fragmentation of impinging molten droplets during thermal spray. Thin Solid Films, 2001, 385(1/2): 132-141.

[36] LI C J, LI J L. Evaporated-gas-induced splashing model for splat 
formation during plasma spraying. Surf. Coat. Tech., 2004, 184(1): $13-23$.

[37] LI H, COSTIL S, LIAO H L, et al. Effects of surface conditions on the flattening behavior of plasma sprayed $\mathrm{Cu}$ splats. Surf. Coat. Tech., 2006, 200(18/19): 5435-5446.

[38] TRAN A T T, HYLAND M M, SHINODA K, et al. Influence of substrate surface conditions on the deposition and spreading of molten droplets. Thin Solid Films, 2011, 519(8): 2445-2456.

[39] MCDONALD A, MOREAU C, CHANDRA S. Effect of substrate oxidation on spreading of plasma-sprayed nickel on stainless steel. Surf. Coat. Tech., 2007, 202(1): 23-33.

[40] PARIZI H B, ROSENZWEIG L, MOSTAGHIMI J, et al. Numerical simulation of droplet impact on patterned surfaces. J. Therm. Spray Techn., 2007, 16(5/6): 713-721.

[41] SHINODA K, RAESSI M, MOSTAGHIMI J, et al. Effect of substrate concave pattern on splat formation of yttria-stabilized zirconia in atmospheric plasma spraying. J. Therm. Spray Techn., 2009, 18(4): 609-618.

[42] KHAN A N, LU J, LIAO H. Effect of residual stresses on air plasma sprayed thermal barrier coatings. Surf. Coat. Tech., 2003, 168(2-3): 291-299.

[43] CHRistoulis D K, PANTELIS D I, DE DAVE-FABREGUE N, et al. Effect of substrate temperature and roughness on the solidification of copper plasma sprayed droplets. Mat. Sci. Eng. a-Struct., 2008, 485(1/2): 119-129.

[44] LI D C, ZHAO H Y, ZHONG X H, et al. Effect of the bond coating surface morphology on ceramic splat construction. J. Therm. Spray Techn., 2015, 24(8): 1450-1458.

[45] SYED A A, DENOIRJEAN A, HANNOYER B, et al. Influence of substrate surface conditions on the plasma sprayed ceramic and metallic particles flattening. Surf. Coat. Tech., 2005, 200(7): 2317-2331.

[46] SALIMIJAZI H R, PERSHIN L, COYLE T W, et al. Effect of droplet characteristics and substrate surface topography on the final morphology of plasma-sprayed zirconia single splats. J. Therm. Spray Techn., 2007, 16(2): 291-299.

[47] LI C J, LI C X, YANG G J, et al. Examination of substrate surface melting-induced splashing during splat formation in plasma spraying. J. Therm. Spray Techn., 2006, 15(4): 717-724.

[48] BROSSARD S, MUNROE P R, TRAN A T T, et al. Study of the splat microstructure, splat-substrate interface, and the effects of substrate heating on the splat formation for ni-cr particles plasma sprayed on to aluminum substrates. J. Therm. Spray Techn., 2010, 19(5): 1115-1130. 